\title{
3D Synthetic Aperture Imaging Using a Water-Jet Coupled Large-Aperture Single Transducer.
}

OF TECHNOLOGY

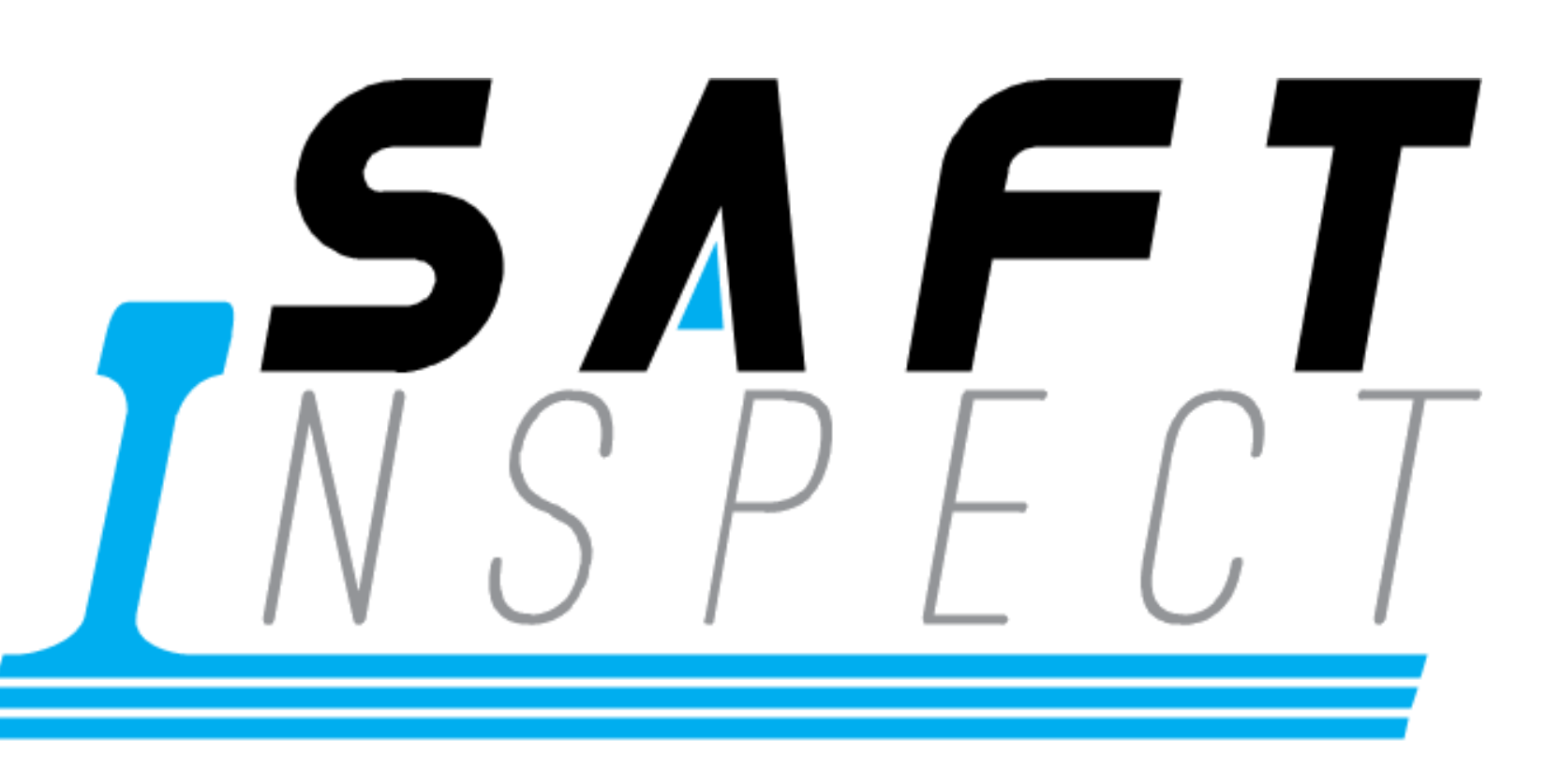

www.saftinspect.eu/

\author{
Miguel Castaño Arranz \\ Johan E. Carlson \\ Matti Rantatalo \\ Robert Risberg \\ Miles Weston
}

\begin{abstract}
Luleå University of Technology
Luleå University of Technology

Luleå University of Technology

Creo Dynamics AB

TWI Technology Centre Wales
\end{abstract}

Abstract: We present a novel technique that uses a transducer that focuses the sound at the surface of the sample, generating a diverging sound field in the sample. The novelty is in successfully applying the Virtual Source (VS) concept using water jet coupling for a large transducer. By focusing the sound field, the water jet probe can be built with a small nozzle opening, limiting the water consumption and making it viable for field applications.

The annular geometry of the large transducer ensures the spherical wavefront assumed in the application of the SAFT algorithm, which usually limits the size of the transducer.

\section{Synthetic Aperture Focusing Technique (SAFT) for ultrasound imaging}

- The traditional fixed focus approach gives the best resolution only at the focal zone.

- SAFT provides a dynamic focusing with uniform resolution.

- In SAFT, a synthetic focus is obtained in the postprocessing of the pulse-echo signals acquired by scanning with a single-element transducer.

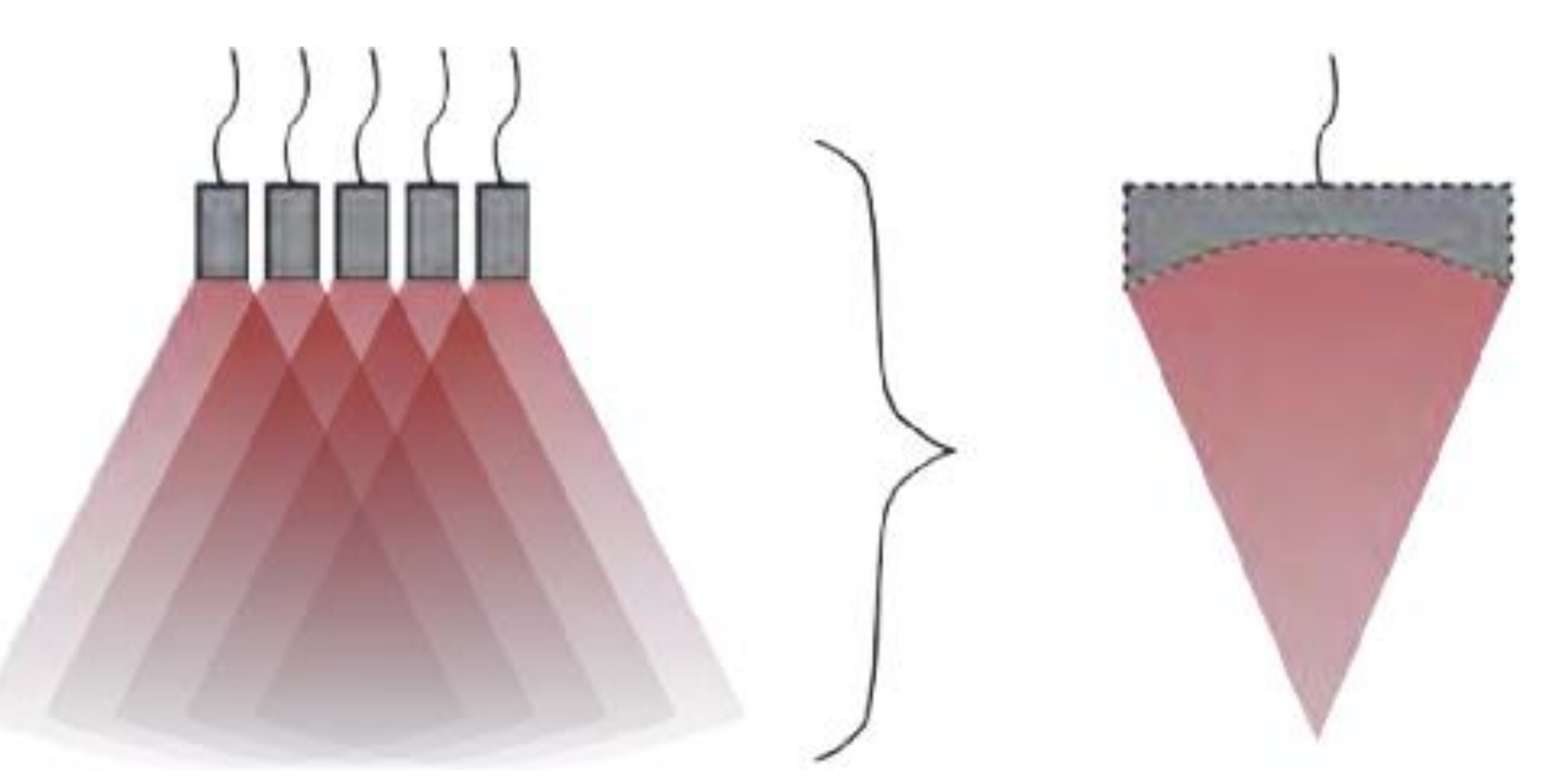

Illustration of the synthetic aperture focusing concept. Figure obtained from [1].

\section{Water-jet coupling with annular transducer array}

- A large transducer improves the SNR but increases the refraction effects at the surface of the material. A solution to mitigate the refraction is focusing the ultrasound beam at the surface of the material (Virtual Source).

- The transducer array has an annular shape to ensure the spherical wavefront required for the application of SAFT.

- A water nozzle has been designed to couple the ultrasound during field tests. The focusing of the ultrasound at the surface allows to build a narrow nozzle with low water consumption.

\section{Case-study on aluminum with side-drilled} holes

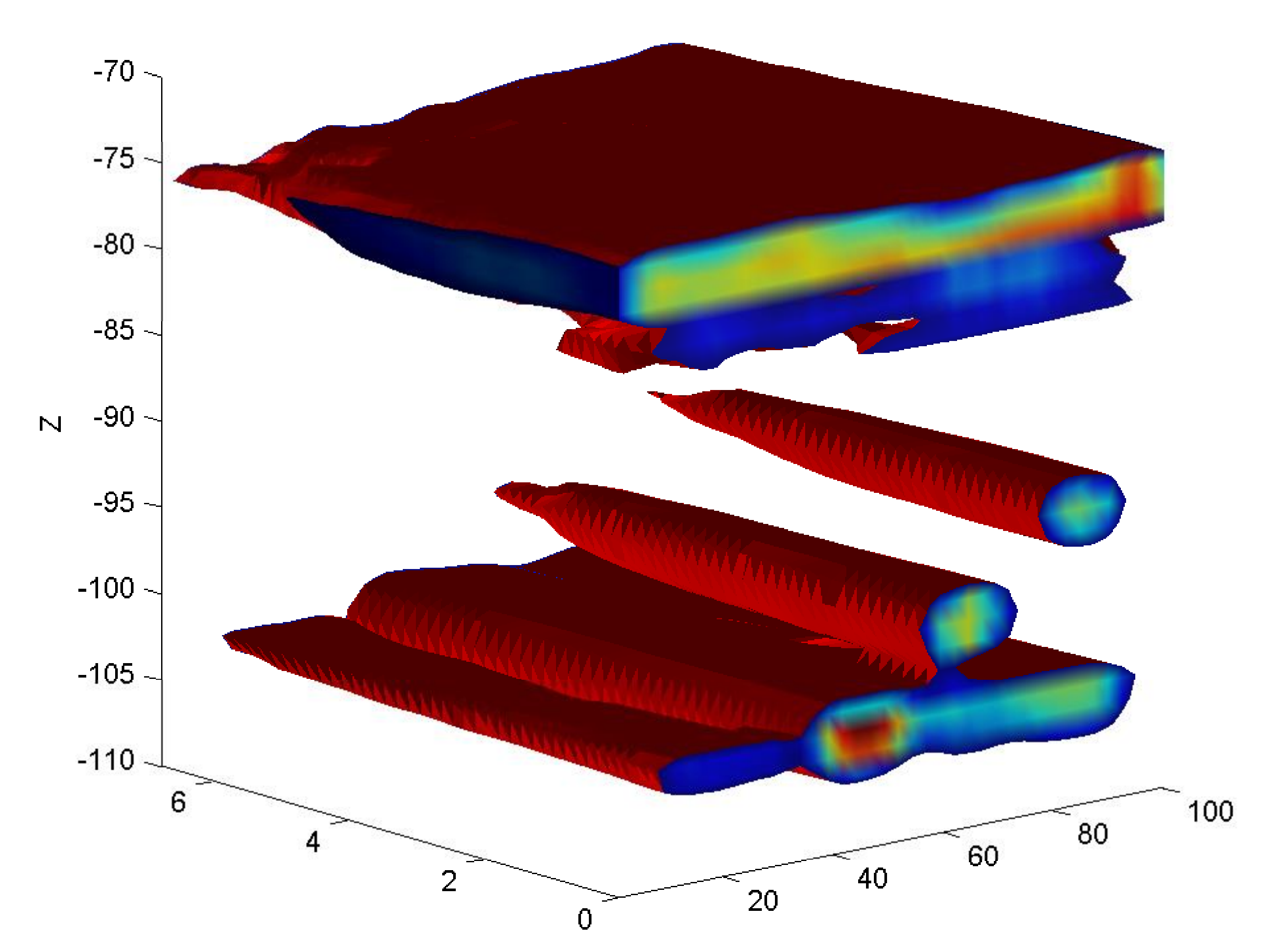

Reconstruction using surface representation.

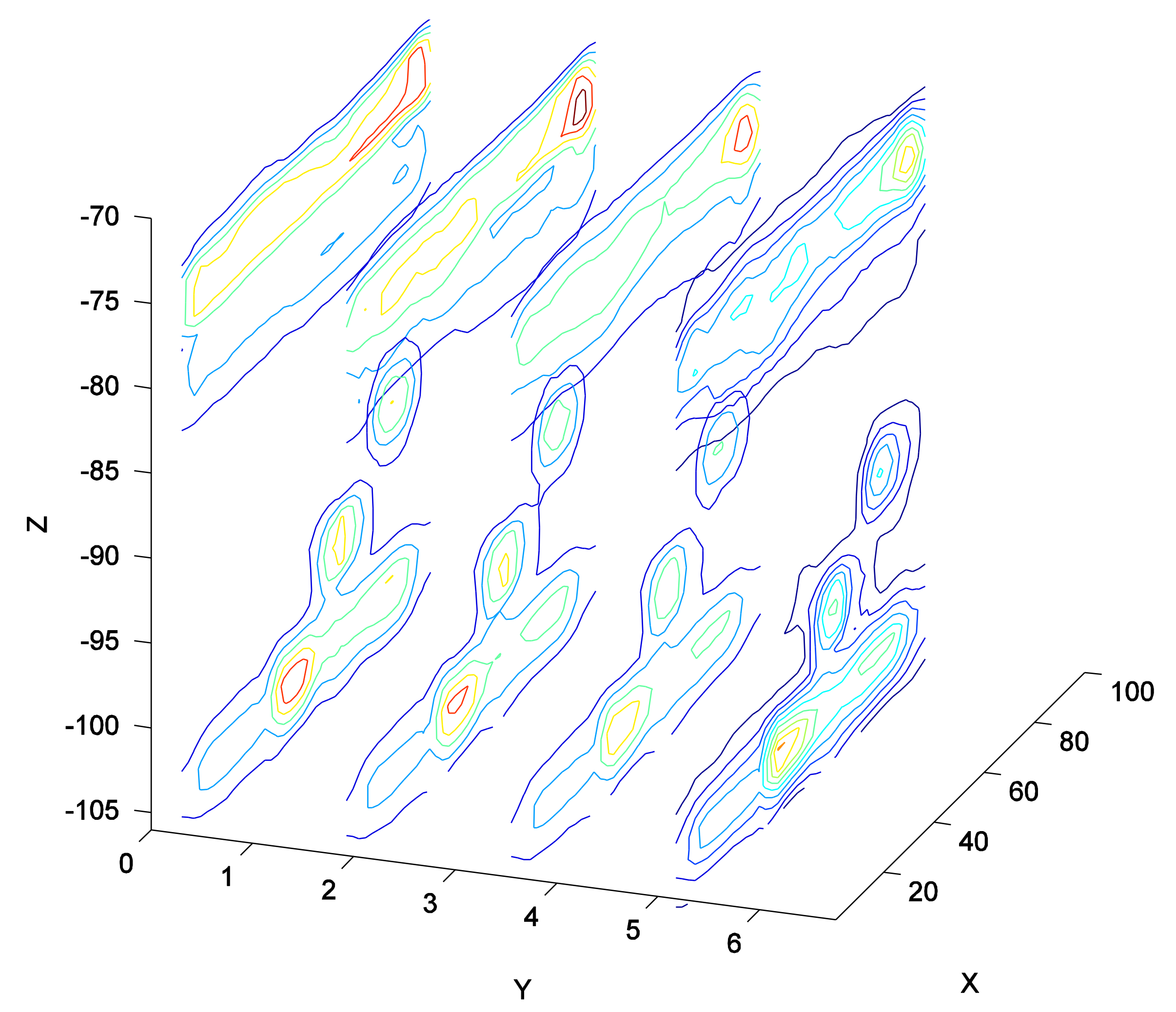

Reconstruction using contour slices.

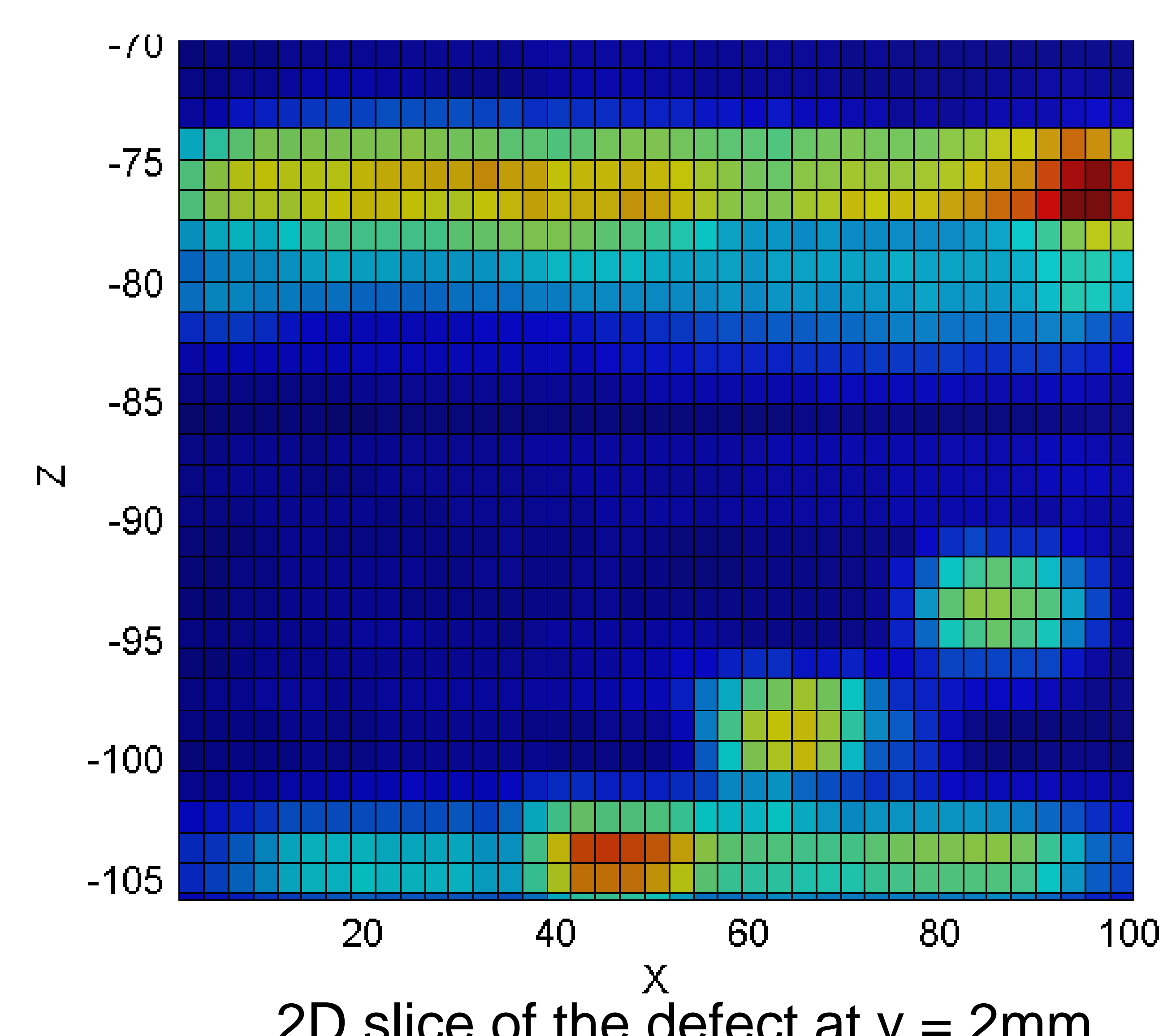

[1] M. H. Skjelvareid, "Synthetic aperture ultrasound imaging with application to interior pipe inspection," Ph.D. Thesis at University of Tromso, 2012.

Contact:

Miguel.Castano@ltu.se Johan.Carlson@ltu.se atti.Rantatalo@ltu.se

\section{Funded by: \\ Coordinator:}

$=$

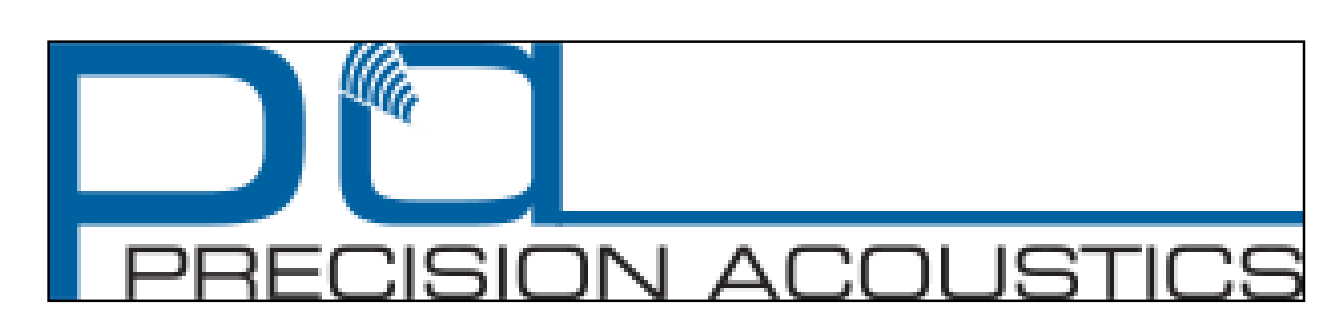

, 